\title{
Summary of: What factors influence the provision of preventive care by general dental practitioners?
}

\section{FULL PAPER DETAILS}

${ }^{1}$ Centre for Values, Ethics and the Law in Medicine School of Public Health; and Population Oral Health Research Unit, Faculty of Dentistry, University of Sydney, Sydney, New South Wales, Australia

Correspondence to: Alexandra Sbaraini Email:alexandra.sbaraini@sydney.edu.au Refereed Paper Accepted 19 April 2012

DOI: 10.1038/sj.bdj.2012.498

${ }^{\circ}$ British Dental Journal 2012; 212: E18

\section{A. Sbaraini ${ }^{1}$}

Background What factors influence a general dental practitioner to offer preventive care to patients? A potential answer to this question is presented based on the findings of a qualitative study recently undertaken in general dental practice in Australia. Method A model of how practices come to be oriented towards preventive or restorative care is described, condensing all of the findings of the study into a single framework. Eight practices were studied and highlighted the interaction between two factors: leadership in practice and prioritisation of cultural, social and economic resources. Results In this model, dentists' leadership to reorient the prioritisation of resources towards preventive care was crucial. Ideally a whole practice changed to preventive philosophy, but change was also possible in a single dentist within a practice. Prioritisation of resources was also key and interacted with dentist leadership. Prioritisation could be seen in the reorganisation of space, routines and fee schedules. During this process, one key support factor for dentists was their external networks of trusted peers and respected practicing dentists. These peers were crucial for transferring preventive knowledge within small networks of dentists who trusted one another; their influence was reportedly more important than centrally produced guidelines or academic advice. In order to help dentists change their practices towards preventive care, the findings from this study suggest that it is important to intervene in these local networks by identifying local dental opinion leaders. During this study, the key conditions needed for practices to reorient to preventive care included the presence of a committed leader with a prevention-supportive peer network, and the reorientation of space, routines and fee schedules to support preventive practice.

\section{EDITOR'S SUMMARY}

What would make you and your team members choose a preventive approach? Despite a large amount of evidence to support non-operative care of early carious lesions the vast majority of dentists are still choosing to pursue a restorative rather than preventive course of action. There is a significant gap between science and practice. But why? What factors might influence the preventive choice?

According to this Australian study of dental practices by Alexandra Sbaraini, leadership and prioritisation of resources within practices were found to be key in implementing preventive techniques. Sbaraini looked at eight practices in Australia where the majority of dentists are independent, self-employed practitioners. These practices implemented preventive protocols and both patients and the dental team were interviewed regarding the experience and outcomes of employing these methods. Some practices followed a structured preventive care regime whereas the 'control' group of practices used a more general preventive approach without performing a caries risk assessment. Questions asked included: 'How easily were you able to implement preventive protocols in this practice?' and 'What did this implementation process entail?'

Transformational leadership is highlighted in the results of the study as imperative in driving a move from restorative to preventive care. The research suggests that the practice leaders must firstly be convinced that a preventive care approach will not result in a drop in practice income. Reorganisation of resources, such as space and fee schedules, towards a preventive philosophy are also required to ensure a successful reorientation.

The facts and the science are there for all to see but the change needs to be made to happen through a shift in culture. The study identifies knowledge transfer and support of trusted dental networks as important in pushing this cultural change towards preventive care. In this age of Web 2.0 and social media there are ample opportunities to meet and exchange ideas and experiences both in the 'real' world and through the internet, eg BDA communities http://community. bda.org/.

Through the evaluation of the experiences of these Australian practices, the changes required to help move your practice towards preventive care are highlighted in this detailed and interesting study.

The full paper can be accessed from the $B D J$ website (www.bdj.co.uk), under 'Research' in the table of contents for Volume 212 issue 11.

Ruth Doherty Managing Editor

DOI: 10.1038/sj.bdj.2012.496 
TO ACCESS THE BDJ WEBSITE TO READ THE FULL PAPER:

- BDA Members should go to www.bda.org.

- Click the 'login' button on the right-hand side and enter your BDA login details.

- Once you have logged in click the 'BDJ' tab to transfer to the BDJ website with full access.

IF YOUR LOGIN DETAILS DO NOT WORK:

- Get a password reminder: go to www.bda.org, click the login button on the right-hand side and then click the forgotten password link.

- Use a recommended browser: we recommend Microsoft Internet Explorer or Mozilla Firefox.

- Ensure that the security settings on your browser are set to recommended levels.

IF YOU HAVE NOT YET SIGNED UP TO USE THE BDA WEBSITE:

- Go to www.bda.org/getstarted for information on how to start using the BDA website.
IN BRIEF

- To inform the reader of the factors that influence a general dental practitioner to offer preventive care to patients.

- To provide a model of how dental practices come to be oriented towards either preventive or restorative care.

- To highlight the resources that dentists require for providing leadership towards preventive care.

\section{COMMENTARY}

'Everybody says that prevention is better than cure, and hardly anyone acts as if they believed it. ${ }^{1}$ The premise of this statement is as pertinent today as it was in 1953 when written by J. M. Mackintosh, professor of public health at the University of London. While community dental public health efforts in the main are preventively focused (community water fluoridation, oral health promotion activities), when it comes to oral health care services themselves, they remain clearly in the realm of repair and reconstruction, not prevention and health maintenance. Who can blame the providers of these services? As Mackintosh continued; 'treatment is more tangible, more immediately rewarding than prevention'. When we provide dental care today, it doesn't seem that there has been a significant shift from the situation identified by Mackintosh. It is enlightening to read, therefore, that there are identifiable, measurable and practical opportunities that might be useful in engineering changes in the manner in which dental practices and individuals within those practices, see the world around them and respond. Activities identified in this study give some hope that support for professionals who may well be wishing to move out of the 'restorative cycle' of practice is possible - and from both their, and the communities perspective, this is a desirable move. 'Palliatives take precedence over prevention, and the health services are overloaded with salvage' noted Mackintosh. ${ }^{1}$ Sounds familiar! Possibilities such as identification of key opinion leaders with transplantation into susceptible dental care environments or the establishment of influential local networks are offered by the authors of this paper. Quite rightly in my view, despite being a necessary precursor, change will not just arise merely from modifying university education. A crucial element lies in those places where the services are provided - in this case, the workplace of the dental practitioner. While many might scoff at such options as being unworkable, the alternative of doing nothing and accepting the patch up path that we've been doggedly following also seems unlikely to succeed - just as it hasn't in the six decades since 1953.

\section{Professor Mike Morgan \\ Melbourne Dental School \\ Faculty of Medicine, \\ Dentistry \& Health Sciences \\ The University of Melbourne}

1. Mackintosh, J M. Trends of Public Opinion about Public Health 1901-51. London: OUP, 1953.

\section{AUTHOR QUESTIONS AND ANSWERS}

1. Why did you undertake this research? Being a dentist and a young researcher, who is aware of the fact that research evidence clearly demonstrates that nonsurgical management of dental caries is efficacious, has instigated me to question why Australian dentists persist with invasive surgical management of enamel and dentine lesions that, if managed non-surgically, would be likely to remineralise. Then, for my $\mathrm{PhD}$ thesis, I conducted a grounded theory study of how evidence-based preventive dental care could be adapted into a group of dental practices and how this process varied between practices. This paper provides an overall explanation based on all findings from my PhD project.

\section{What would you like to do next in this} area to follow on from this work?

Translating research findings into dental practice is an important research topic. Dental care is expensive and providing treatments that are not based on evidence is a waste of resources and can be potentially harmful to patients. In a future project I would like to assess the factors that influence whether dentists will change their clinical behaviour according to research evidence in general and potentially develop new ways to support the translation of evidencebased dental practice in Australian and international general practice. 